\title{
Revealing the Role of Anchoring Groups in the Electrical Conduction Through Single-Molecule Junctions
}

\author{
Linda A. Zotti, Thomas Kirchner, Juan-Carlos Cuevas, Fabian Pauly, Thomas Huhn, \\ Elke Scheer, and Artur Erbe*
}

\begin{abstract}
A combined experimental and theoretical study is presented revealing the influence of metal-molecule coupling on electronic transport through single-molecule junctions. Transport experiments through tolane molecules attached to gold electrodes via thiol, nitro, and cyano anchoring groups are performed. By fitting the experimental current-voltage characteristics to a single-level tunneling model, we extract both the position of the molecular orbital closest to the Fermi energy and the strength of the metal-molecule coupling. The values found for these parameters are rationalized with the help of density-functional-theory-based transport calculations. In particular, these calculations show that the anchoring groups determine the junction conductance by controlling not only the strength of the coupling to the metal but also the position of the relevant molecular energy levels.
\end{abstract}

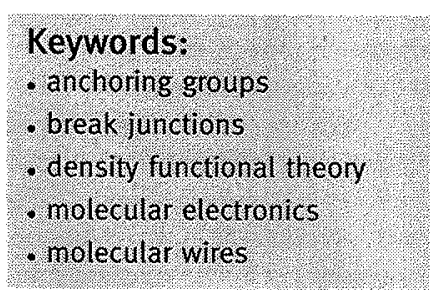

[*] Dr. A. Erbe, ${ }^{\ddagger}$ T. Kirchner, ${ }^{\dagger} \S$ Prof. E. Scheer Physics Department, University of Konstanz 78457 Konstanz (Germany)

E-mail: a.erbe@fzd.de

Dr. L. A. Zotti, ${ }^{,}$Prof. J.-C. Cuevas

Departamento de Física Teórica de la Materia Condensada Universidad Autónoma de Madrid 28049 Madrid (Spain)

Dr. F. Pauly

Institut für Theoretische Festkörperphysik Karlsruhe Institute of Technology (KIT)

76131 Karlsruhe (Germany)

Dr. T. Huhn

Chemistry Department, University of Konstanz 78457 Konstanz (Germany)

[t] Current address: Forschungszentrum Dresden-Rossendorf 01328 Dresden (Germany)

[§] Current address: Institute for Biomedical Engineering University of Zurich and ETH Zurich 8092 Zurich (Switzerland)

[t] These authors contributed equally to the work.

Supporting Information is available on the WWW under http:// www.small-journal.com or from the author.

\section{Introduction}

In the past few years, the development of a variety of nanofabrication techniques has allowed individual molecules to contact between metallic electrodes and to measure their electronic transport properties. ${ }^{[1,2]}$ These techniques have also made it possible to show that certain molecules can indeed behave like some of the basic components of today's microelectronics, which has given rise to the field of molecular electronics. ${ }^{[3]}$ Although the initial results are promising, the future of this field still depends crucially on our ability to understand the transport mechanisms that determine the electrical current at the single-molecule level. In this respect, great effort has recently been devoted to understand how the transport properties of molecular junctions can be tuned by chemically modifying the molecules under study. ${ }^{[4]}$

One of the main problems that remains to be understood is the role of anchoring groups in the transport through molecular junctions. ${ }^{[5-13]}$ Different experiments have shown that the terminal groups that bind a molecule to the metal electrodes not only control the numerical value of the junction conductance but also affect several other factors. Thus, for instance, Venkataraman et al. ${ }^{[8]}$ have shown that measurements with amine-anchored molecules exhibit a considerably lower spread of the conductance values than, for example, measurements with their thiolated partners. The anchoring groups have also 
been shown to infuence the exponential decay of the conductance with molecular length ${ }^{[9]}$ as well as the length over which a junction can be stretched ${ }^{[11]}$ Furthermore, terminal groups can also be used to tune the current rectification in a junction ${ }^{[12]}$ and even to modify the dominant transport mechanism. ${ }^{[13]}$

From the theory side, extensive studies have been carried out in order to elucidate the origins of the observations detailed above. For instance, it has been shown that the conductance of thiolated molecules is quite sensitive to the binding geometry ${ }^{[1-16]}$ while the better defined conductance values of amineterminated compounds have been explained in terms of the preference of this group to bind selectively to low-coordinated gold atoms. ${ }^{[8,17-19]}$ It has been argued that the anchoring groups affect the energy-level line up relative to the metal Fermi energy, and consequently the conductance, ${ }^{[20]}$ and it has also been suggested that they can play an important role in determining both the length of the conductance plateaus and the force required to break the contacts. ${ }^{[9.11]}$

With the notable exception of recent thermopower measurements, ${ }^{[21,22]}$ the experimental studies on the role of anchoring groups have been based on the analysis of the lowbias conductance, which does not provide spectroscopic information to elucidate how these groups exactly modify the electronic structure of molecular junctions. Presently, a systematic study that correlates the conductance values, binding energies, and coupling strengths with the chemistry of the anchoring groups is still missing. In this Full Paper, we present a combined experimental and theoretical analysis of the transport properties of 1,2-bisphenylethene (tolane) molecules, which were chemically modified by the inclusion of thiol, nitro, and cyano anchoring groups. As is shown below, the analysis of the current-voltage $(I-V)$ characteristics of this testbed molecule allows us to quantitatively establish how these different anchoring groups determine the metal-molecule coupling and how they influence the position of the molecular levels, altering in turn the nature of the electrical conduction.

\section{Results and Discussion}

\subsection{Measurements of $I-V$ curves}

In this Full Paper, we present measurements on metalmolecule-metal junctions, which were produced using the mechanically controlled break-junction (MCBJ) technique (see Experimental Section for details). The main advantage of our experimental setup is its high mechanical stability, even in liquid environments, which allows us to measure $I-V$ characteristics at all stages of the opening and closing curves. This, however, distorts the typical conductance-distance traces. ${ }^{[24,25]}$ Thus, an analysis of these curves in terms of histograms is not presented in this work but we investigate instead the statistics of the $I-V$ curves. In general, we can distinguish three regimes during the opening and closing cycles: i) After the direct contact of the last metal atoms is broken, pure metal-to-metal tunneling is observed. The distance between the metallic electrodes is still so small that the tunneling current exceeds any contribution by transport through the molecules. This regime is characterized by linear $I-V$ characteristics in the whole range from $-V_{\max }$ to
$V_{\max }$.ii) When the electrodes are pulled further apart, the metal-to-metal tunneling contribution decreases exponentially and then most of the current flows through the molecules. In this regime, the $I-V$ curves are nonlinear, as can be seen in Figure 1 in which some examples for the three different molecules are shown. iii) Further separation of the electrodes leads to loss of contact between the metallic electrodes and the molecules. In this case, the current flows via tunneling between the metal electrodes, mediated by the solvent. ${ }^{[26]}$ The current is generally far below the level that is observed in the two previous cases. Let us mention that hysteretic effects in the $I-V$ curves can be observed, which we attribute to the presence of the solvent. The current, however, is generally so low that these effects do not play a significant role in regimes (i) and (ii).

Motivated by the analysis of the different types of $I-V$ curves, we focus our attention here on the study of junctions with values of the low-bias conductance between $10^{-1}$ and $10^{-5}$ $G_{0}$ (with $G_{0}=2 e^{2} / h$ ), which are likely due to either a single molecule or a small number of them. Since the contact can be realized in this regime by many geometrical configurations, we carry out a statistical analysis of the $I$-V curves. ${ }^{[27]}$ Obviously, the $I-V$ characteristics contain more information than just the linear conductance. Thus, for instance, we find that junctions with similar linear conductance values can give rise to very distinct $I-V$ curves. This illustrates that the more conventional analysis of histograms to identify the most probable value of the conductance of a single-molecule contact ${ }^{[4,6-11,28,29]}$ certainly misses important information about the molecular-transport junctions.

A first inspection of the $I-V$ curves for the different anchoring groups (see Figure 1) reveals that while the currents for the $4,4^{\prime}$-biscyanotolane (BCT) molecules (cyano group) are on the order of $1 \mathrm{nA}$ for a voltage of $0.5 \mathrm{~V}$, the corresponding currents for 4,4'-bisthiotolane (BTT; thiol group) and 4,4'bisnitrotolane (BNT; nitro group) are typically around $1-10 \mu \mathrm{A}$ (i.e., more than three orders of magnitude higher). Moreover, the number of stable $I-V$ curves that were recorded for the $\mathrm{BCT}$ molecules was clearly much smaller than for the other two, which we attribute to the weak metal-molecule coupling in the case of this end group (see discussion below). A comparison of the results for BTT and BNT shows that the nitro group leads to higher currents than in the case of thiol, especially at low bias.

\subsection{Single-Level Model}

In order to obtain an insight into the physical origin of these results, it would be very helpful to be able to reproduce the $I-V$ curves with a simple theoretical model. Our choice is a singlelevel model, which is often referred to as resonant-tunneling model. ${ }^{[30]}$ This model has already been used, for instance, to explain transport through $\mathrm{C}_{60}$-based molecules, ${ }^{[31]}$ to describe transition-voltage spectroscopy data on alkanes of various length, ${ }^{[32]}$ or to fit the $I-V$ curves in molecular junctions fabricated with on-wire lithography. ${ }^{[33]}$ The main assumptions for the validity of the model are: (i) the transport is phase coherent (tunneling mechanism) and (ii) the current is dominated by a single molecular resonance in the entire voltage range explored in the experiments. While the first assumption is very reasonable for short molecules like the tolane studied here, 

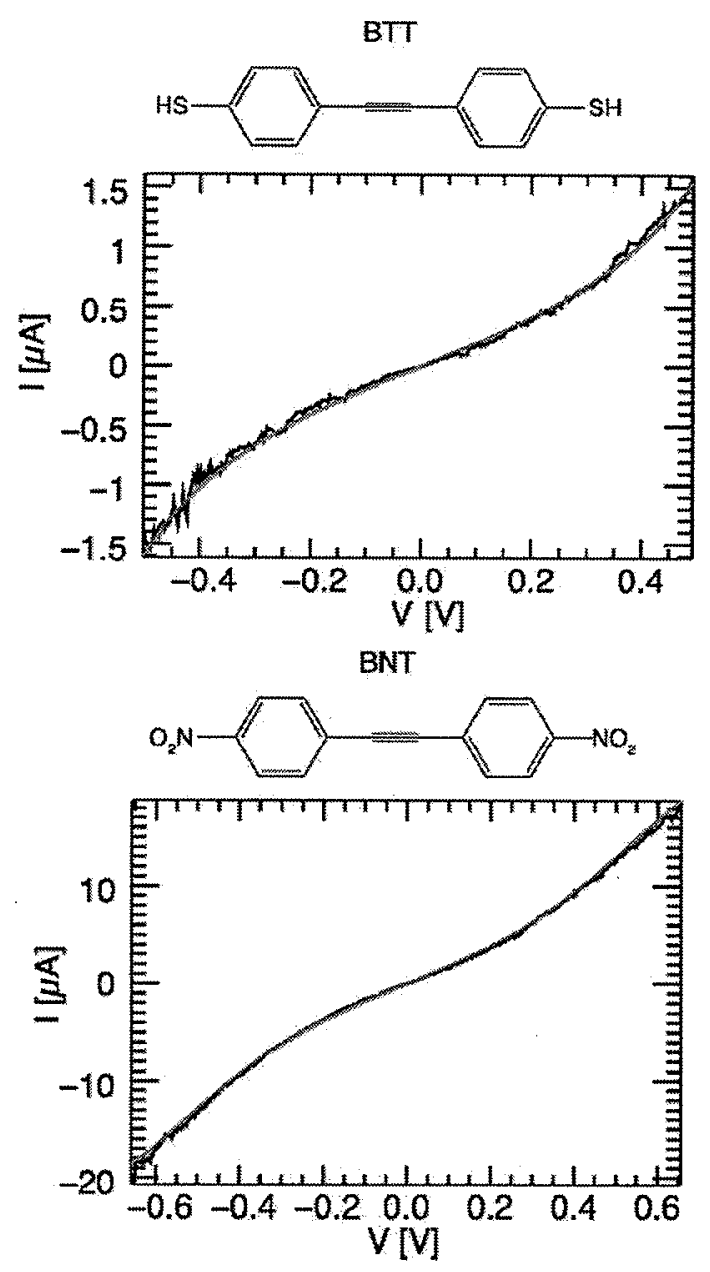

it is not obvious that the second one is justified (see discussion in Section 2.3.).

Within this model, the current through a metal-moleculemetal junction is given by the Landauer formula, ${ }^{[30]}$

$I(V)=\frac{2 e}{h} \int_{-\infty}^{\infty} T(E, V)[f(E-e V / 2)-f(E+e V / 2)] d E$

where $T(E, V)$ is the transmission function that depends both on the energy $E$ and voltage $V$ and $f(E)=1 /[1+\exp ((E-\mu) /$ $\left.k_{\mathrm{B}} T\right)$ ] is the Fermi function with $\mu$ as the lead chemical potential and $k_{\mathrm{B}} T$ being the thermal energy. In this model, the transmission is given by the Breit-Wigner formula: ${ }^{[30]}$

$T(E, V)=\frac{4 \Gamma_{\mathrm{L}} \Gamma_{\mathrm{R}}}{\left[E-E_{0}(V)\right]^{2}+\left[\Gamma_{\mathrm{L}}+\Gamma_{\mathrm{R}}\right]^{2}}$

Here, $E_{0}$ is the energy of the molecular level, which can depend on the bias voltage, and $\Gamma_{\mathrm{L} / \mathrm{R}}$ are the scattering rates related to the probability of injecting an electron into the molecule from the left $(L)$ and right $(R)$ electrodes. These rates also describe the width (or inverse lifetime) that the molecular level acquires in virtue of the coupling to the metal and they can be used as a measure of the strength of the metal-molecule coupling. Furthermore, we shall assume that the rates are energy and voltage independent, which is a good approximation for the case of a metal like gold with a rather flat density of states in the vicinity of the Fermi energy. Additionally, we assume that the voltage drops at the metal-molecule interfaces and that the relative drop is determined by the ratio $\Gamma_{\mathrm{L}} / \Gamma_{\mathrm{R}}$. This means, in particular, that in the case of symmetric junctions $\left(\Gamma=\Gamma_{\mathrm{L}}=\Gamma_{\mathrm{R}}\right), E_{0}$ does not depend on the bias and the $I-V$ curves are symmetric with respect to voltage inversion (i.e., $I(-V)=-I(V))$.

We have used the single-level model summarized in Equations 1 and 2 to fit the experimental $I-V$ curves. We concentrate here on symmetric $I-V$ curves in order to reduce the number of fitting parameters to two, namely $\Gamma$ and $E_{0}$, and thus simplify their extraction. It is worth stressing that asymmetric curves arise for all tested molecules. They can also be described successfully by the single-level model assuming coupling parameters where $\Gamma_{\mathrm{L}} \neq \Gamma_{\mathrm{R}}$ (see Supporting Information). Both the energy levels and coupling constants are very similar to those obtained from the symmetric curves. As can be seen in Figure 1, the model is able to reproduce the experimental data very satisfactorily. The values extracted for $\Gamma$ and $E_{0}$ (which we always measure with respect to the equilibrium Fermi energy, $E_{\mathrm{F}}$ ) are summarized in Figure 2 for the three molecules studied. The changes in the parameters refiect the different configurations adopted by the molecular

Figure 1. Molecules investigated: $B T$ (top), BNT (center), and BCT (bottom) and typical symmetric $I-V$ characteristics measured for junctions with the three molecules under study. The curves were recorded by sweeping the voltage from $0 \mathrm{~V}$ to $0.7 \mathrm{~V}$, then to $-0.7 \mathrm{~V}$, and back to $0 \mathrm{~V}$. For clarity, only the sweep from $0.7 \mathrm{~V}$ to $-0.7 \mathrm{~V}$ is shown (black curve). For the example shown in the top panel, the maximum voltage was $V_{\max }= \pm 0.5 \mathrm{~V}$ only. The red curve shows the fit to the single-level model The parameters used in the fits were: $\Gamma=43 \mathrm{meV}, E_{0}=0.45 \mathrm{eV}$ for BT, $\Gamma=70 \mathrm{meV}, E_{0}=0.27 \mathrm{eV}$ forBNT, and $\Gamma=0.85 \mathrm{meV}, E_{0}=0.54 \mathrm{eV}$ for BCT. $E_{0}$ does not depend stror following: 1) For BTT, $E_{0}$ does not depend strongly on the parameter $\Gamma$ increases on average with increasing current level from 20 to $100 \mathrm{meV}$. ii) For BNT, the values of the parameters are similar to those for BTT but they exhibit a larger spread. Notice also that we find a slightly higher current level for BNT 

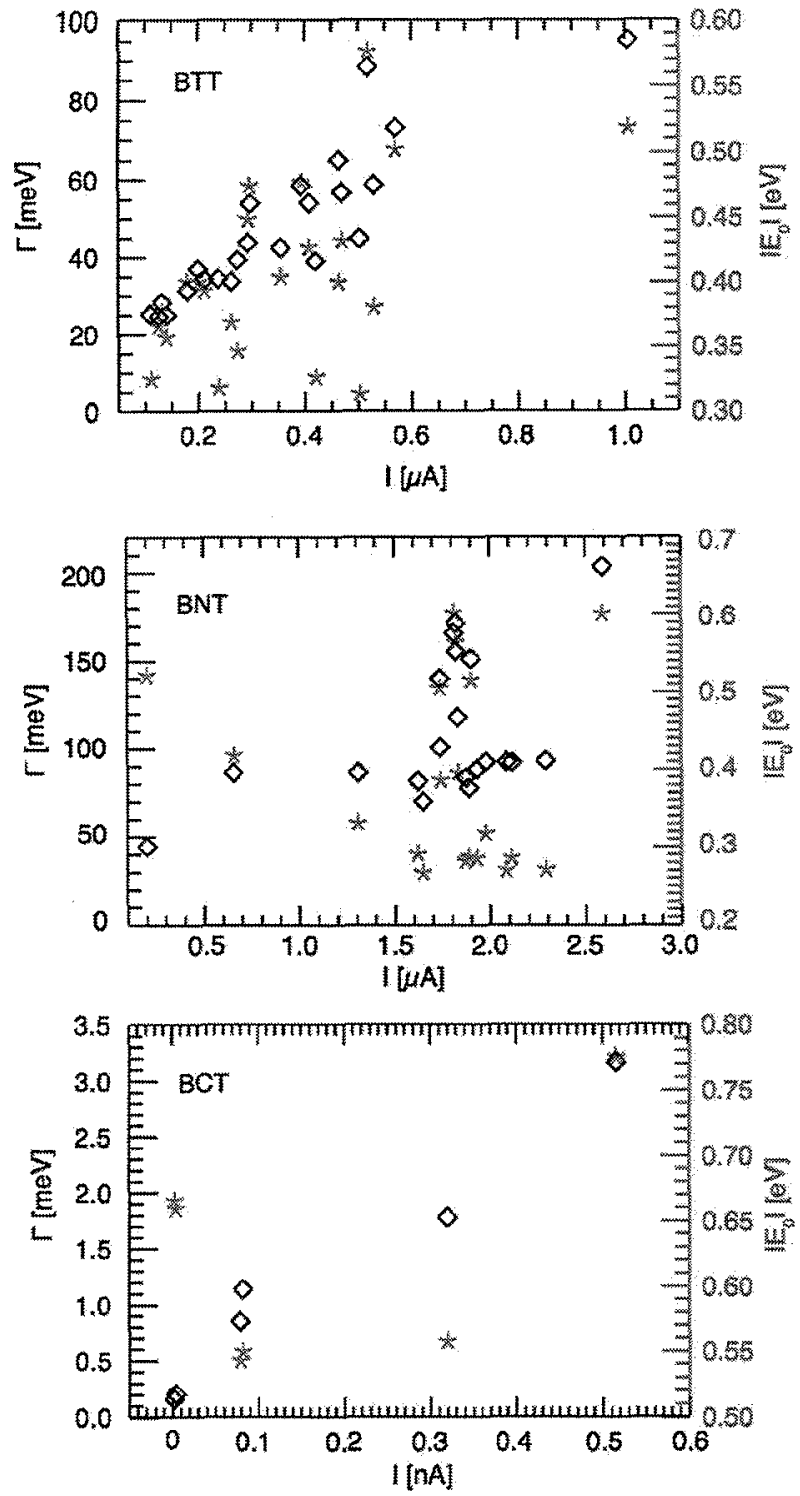

Figure 2. Coupling parameter $\Gamma$ (black diamonds) and absolute value of the level position $\left|E_{o}\right|$ (red stars) extracted from the fits to the $I-V$ curves of the three molecules (BT, BNT, and $B C T$ ) as a function of the current at $V=100 \mathrm{mV}$. The data are taken from all the $l-V$ curves that were symmetric.

as compared with BTT and also higher values for the coupling parameter $\Gamma$ (between 50 and $200 \mathrm{meV}$ ). In addition, we find no clear tendency for the parameters as a function of the current. iii) The results for $\mathrm{BCT}$ show that the coupling to this molecule is much weaker $(\Gamma \approx 1-3 \mathrm{meV})$ and that the energy level is further away from the Fermi energy ( $E_{0}$ between 0.5 and $0.8 \mathrm{eV})$. The coupling tends to increase with increasing current, similar to the behavior for thiol anchors.

It is worth stressing that, in all the cases reported in Figure 2, we are dealing with (moderate) off-resonant tunneling through a single level. In the symmetric situation that we are discussing here, the resonant condition is reached at a voltage $V=2 E_{0} / e$, which should be manifested as a rapid increase of the current at that voltage. However, in our measurements, the lowest values for $\left|E_{0}\right|$ are about $0.3 \mathrm{eV}$, whereas the maximum applied voltages range from 0.5 to $0.7 \mathrm{~V}$. Thus, the resonant condition is only reached in our measurements for particular junctions, that is, when the junctions were stable up to a bias voltage $V>2 E_{0} / e$. In such cases the overall conductance is rather high and the increase in current due to the resonance condition is difficult to detect. A typical curve for this scenario is shown in Figure 2 for the case of the BNT molecule. For this reason, we prefer to call the model introduced in this section a single-level rather than resonant-tunneling model.

\subsection{Density Functional Theory Calculations}

The results presented above provide a novel way to quantify the role of anchoring groups. However, one needs to be cautious because the $I-V$ curves (see Figure 1) do not have sufficiently pronounced structure as to unambiguously exclude the use of other tunneling models (a discussion of trials to fit the results with other models is presented in the Supporting Information). In this sense, it is very important to justify the assumptions made in the single-level model with first-principle calculations, which in turn should shed light on the origin of the parameter values found in the fits. For this reason, we have carried out calculations based on density functional theory (DFT) to determine the geometry and electronic structure of the molecular junctions as well as their transmission. To be precise, we have first performed DFT calculations using TURBOMOLE ${ }^{[34]}$ in order to describe the molecules in the gas phase and the goldmolecule-gold junctions. In all the calculations presented in this work, we have used a split-valence basis set with polarization functions for all nonhydrogen atoms ${ }^{[35]}$ and the BP86 exchange-correlation functional. ${ }^{[36]}$ To determine the structure of the molecular junctions, geometrical optimizations were performed for systems where the molecules were attached to gold clusters consisting of 20 atoms. Then, further gold layers were added to the optimized structures in order to extend the size of both clusters to around 60 atoms and to describe correctly the metal-molecule charge transfer and the corresponding level alignment. Finally, single-point calculations were performed on these extended systems and the zero-bias transmission of the junctions was computed in the spirit of the Landauer formalism using Green's function techniques, as detailed in Reference [37]. For the sake of completeness, we have also analyzed theoretically the transport through the 1,2 bisaminotolane (BAT), which has been investigated in recent experiments. ${ }^{[38]}$

In Figure 3, we show the computed zero-bias transmission as a function of energy for BTT, BAT, BNT, and BCT molecules in different binding geometries. In the case of BTT, we have considered top and hollow binding positions. For both geometries, the conduction takes place through the highest occupied molecular orbital (HOMO). The transmission resonance is narrower for the hollow geometry and appears at a lower energy value. Concerning the amine group, only the top geometry is shown here because, in agreement with previous calculations, ${ }^{[8,17-19]}$ we find that this group prefers to bind to low-coordinated gold atoms. Also, in this case, the conductance is dominated by the HOMO of the molecule. On the other hand, 


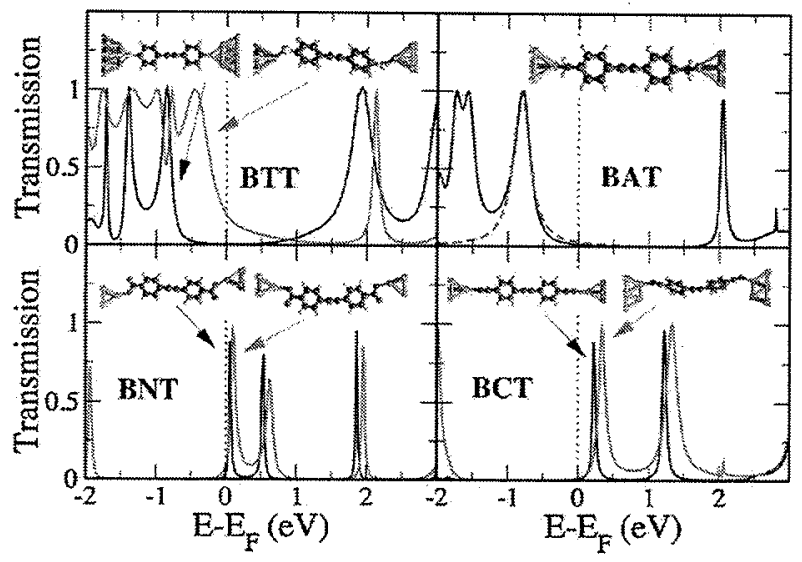

Figure 3. Transmission as a function of energy for different contact geometries and the four molecules considered theoretically. The dashed line in the upper right panel corresponds to the fit of the transmission resonance closest to the Fermi level with the single-level model of Equation 2.

the nitro group was found to bind to gold through the oxygen atoms. $\mathrm{Au}-\mathrm{NO}_{2}$ binding has been suggested by recent experiments ${ }^{[39]}$ and computational studies employing nitro-Au junction were reported recently. ${ }^{[40]}$ For the $\mathrm{Au}-\mathrm{BNT}-\mathrm{Au}$ junctions, we consider a trans and a cis structure, both in top binding positions. These two structures exhibit slightly different transmission functions. The reason is that, in the cis configuration, the molecule is bent in such a way that the two oxygen atoms get closer to the Au cluster. This causes the relevant transmission resonance to be broader, as shown in Figure 3. In both cases, however, the conductance is dominated by the lowest unoccupied molecular orbital (LUMO). For BCT, top and hollow geometries were studied, but one of the hollow bindings changed to a top one, with the molecule adopting a bent structure. Similar to BNT, the conductance is mainly determined by the LUMO for both configurations and the bending leads also in this case to a broader resonance.

The most important thing to notice is that, in all the cases shown in Figure 3, the transport is indeed dominated by a single level, namely the one closest to the gold Fermi level. Moreover, in all those cases it was possible to fit the transmission resonances close to the Fermi energy with Equation 2 for a symmetric case $\left(\Gamma=\Gamma_{\mathrm{L}}=\Gamma_{\mathrm{R}}\right)$ in a certain energy window, which justifies the use of the single-level model (an example of the fit is shown as a dashed line in Figure 3 for the case of the amine group). The obtained values for $E_{0}$ and $\Gamma$ for the geometries of Figure 3 are summarized in Table 1 . The computational results for BTT reproduce the order of magnitude found in the experiments well for both fit parameters. In the case of $\mathrm{BCT}$, we find a smaller value of $\Gamma$ than for BTT, also in agreement with the experimental fits, and also a smaller $E_{0}$ value. For the amine group (BAT), we obtain values for the coupling strength on the order of those found for BTT, while the conductance is lower than for this latter molecule. For the particular geometry shown in Figure 3, the linear conductance is still higher than the experimental value found in Reference [38] $\left(5.7 \times 10^{-4} G_{0}\right)$. The most problematic case is BNT, where the theoretical values for $\Gamma$ are clearly smaller than the experimental ones. More importantly, we have found in this case a binding geometry in which the transport is at least determined by two molecular orbitals. In this geometry (see Supporting Information), the molecule binds to the electrodes via two $\mathrm{Au}-\mathrm{O}$ bonds on each side. In this case, we have found that the LUMO and LUMO +1 of the molecule contribute significantly to the conductance and that the $I-V$ curve is rather linear, similar to the experimental results. This result suggests that, for this molecule, the use of a single-level model might not be justified in general.

As mentioned above, an important piece of information revealed by our calculations is that the nature of the transport depends crucially on the anchoring group. While for BTT and $\mathrm{BAT}$, we find that the transport is dominated by the HOMO (hole transport), in the case of BNT and BCT, the conduction takes place through the LUMO (electron transport). Although the exact positions of the molecular orbitals in the junction depend on the interaction with the metallic electrodes, these findings can be traced back to the electronic structure of the isolated molecules (in the gas phase). In Table 1; we report the position of the frontier orbitals (HOMO and LUMO) for the four different molecules, as obtained from our DFT calculations. These values have to be compared with those of the reference molecule (with no anchoring groups): $-0.374 \mathrm{eV}$ for the HOMO and $+3.652 \mathrm{eV}$ for the LUMO (measured with respect to the gold Fermi energy, which in our calculations is $-5.0 \mathrm{eV}$ ). From these results, one can see that both thiol and amine groups have electron-donating character, which is reflected in the increase of the energy of the frontier molecular orbitals. This results in practice in the fact that the HOMO is the orbital closest to the Fermi energy in the junctions. On the

Table 1. Theoretical results for the HOMO and LUMO energies of the isolated molecules, contact geometries considered in Figure 3 , linear conductance $G$, level position $E_{0}$, and coupling strength $\Gamma$ for the different molecular junctions. The HOMO, LUMO, and $E_{0}$ are measured with respect to the gold Fermi energy, which is $E_{\mathrm{F}}=-5.0 \mathrm{eV}$. For BCT, "top low c." means low coordination of the top gold atom (straight geometry), while "top high c." corresponds to high coordination of this atom (bent geometry). For the sake of comparison, the last two columns show the range of the experimental values for $\left|E_{\mathrm{o}}\right|$ and $\Gamma$ taken from Figure 2 .

\begin{tabular}{|c|c|c|c|c|c|c|c|c|}
\hline Molecule & HOMO [eV] & LUMO $[\mathrm{eV}]$ & Contact geometry & $G\left(G_{0}\right)$ & $E_{0}[\mathrm{eV}]$ & $\Gamma[\mathrm{meV}]$ & $\left|E_{0}\right|[\mathrm{eV}]$ Exp. & $\Gamma[\mathrm{meV}] \operatorname{Exp}$ \\
\hline \multirow[t]{2}{*}{$\mathrm{BTT}$} & 0.075 & 2.689 & top & 0.2 & -0.46 & 110 & $0.3-0.6$ & $20-100$ \\
\hline & & & hollow & 0.0064 & -0.86 & 40 & & \\
\hline BAT & 0.798 & 3.509 & top & 0.014 & -0.81 & 70 & - & - \\
\hline \multirow[t]{2}{*}{ BNT } & -1.435 & 0.965 & trans & 0.03 & 0.06 & 8 & $0.25-0.6$ & $50-200$ \\
\hline & & & cis & 0.083 & 0.11 & 20 & & \\
\hline \multirow[t]{2}{*}{$\mathrm{BCT}$} & -1.197 & 1.411 & top low $c$. & 0.0057 & 0.23 & 10 & $0.5-0.8$ & $1-3$ \\
\hline & & & top high $c$. & 0.013 & 0.34 & 30 & & \\
\hline
\end{tabular}


contrary, nitro and cyano groups have an electron-withdrawing character, which means that the LUMO is pushed closer to the gold Fermi energy. Recent experiments on the thermopower of cyano-terminated benzene molecules did indeed show that the transport in this case is dominated by the LUMO of the molecule ${ }^{[21]}$ and this has also been suggested by theoretical calculations. ${ }^{[20]}$ Thus, from this analysis we can conclude that, for short molecules at least, the anchoring groups determine the transport characteristics of a molecular junction not only by controlling the strength of the metal-molecule coupling but also via their strong influence on the position of the frontier orbitals.

The last issue that we want to address briefly is the dependence of $\Gamma$ on stretching of the contacts. The experimental results of Figure 2 show that the level broadening does not change significantly from realization to realization of the contact (just within a factor of $3-4$ ). This might be surprising at first glance since some of the $I-V$ curves may correspond to contacts subjected to strong stress. In order to clarify this issue, we have performed DFT simulations of the stretching process for all the molecules in the top binding geometries shown in Figure 3. As discussed in detail in the Supporting Information, we find that for all the molecules, the coupling parameter $\Gamma$ only changes within a factor 3 to 5 from the equilibrium geometry to the point at which the contacts break, in accordance with the variation range found experimentally.

\section{Conclusions}

In summary, we have experimentally investigated electron transport through single tolanes modified with different anchoring groups and attached to gold contacts. Using a simple single-level model, we have been able to fit the observed $I-V$ curves, which allows us to extract the position and broadening of the molecular levels that dominate the transport. In addition, we have presented DFT-based calculations that justify the use of this simple tunneling model and provide additional support for the values extracted from the fits. These calculations reveal that the anchoring groups have a strong impact on the position of the relevant molecular orbitals and that they control the nature of the conduction according to their donor or acceptor character.

\section{Experimental Section}

In this work, we employed the $M C B]^{[23]}$ technique to build metalmolecule-metal contacts. The MCBIs were defined lithographically using bronze substrate, a polyimide insulation layer, and $80 \mathrm{~nm}$ of gold on top. Measurements were carried out at room temperature using a refillable glass pipette with a poly(dimethylsiloxane) gasket as a liquid cell, which was lowered onto the break junction. Contact to the Au break-junction structure was established via in-capped Au spring contacts and a computercontrolled Keithley 6430 source measurement unit was used to determine the conductance. Slow opening and closing of the MCB) in the presence of molecules allowed us to record conductance- distance traces. Alternatively, $I-V$ characteristics were taken at fixed sample opening, slowly ramping the voltage from 0 to $V_{\max }$, from $V_{\max }$ to $-V_{\max }$, and back to 0 up to three times, with $V_{\max }$ between 0.5 and $1 \mathrm{~V}$. In order to ensure statistically relevant information, the $I-V$ curves were taken from different opening and closing cycles, in which new Au-molecule-Au contacts were formed each time.

As mentioned above, we chose tolane as a simple conjugated backbone, modified with three different anchoring groups, as shown in Figure 1: BCT with cyano groups, where the $\mathrm{N}$ atom has a lone electron pair, BNT with nitro groups, where the strongly electronegative $\mathrm{O}$ atoms withdraw much of the electron density from the $N$, and BTT with the more standard thiol groups. All these molecules were dissolved in toluene. In the case of BT, a droplet of ammonium solution was added prior to the measurement to facilitate the removal of the acetyl protective groups initially attached to the $\mathrm{S}$ atom.

\section{Acknowledgements}

We gratefully acknowledge the support of U. Eberlein and S. Verleger with the MCBJ setup and thank S. P. Liu and J. Grebing for help with the sample preparation. We are indebted to 1. Wolf for assistance in synthesizing the molecules and $M$. Bürkle and S. Bilan for help with the DFT calculations. We thank the Baden-Wurtemberg Foundation for providing scientific exchange within the research network Functional Nanostructures. L.A.Z. and J.C.C. were funded by the EU through the network BIMORE (MRTN-CT-2006-035859). F.P. acknowledges funding through a Young Investigator Group and the DFG and A.E. through the DFG individual project ER341/2.

[1] N. J. Tao, Nat. Nanotechnol. 2006, 1, 173.

[2] K. Moth-Poulsen, T. Bjørnholm, Nat. Nanotechnol. 2009, 4, 551.

[3] introducing Molecular Electronics (Eds: G. Cuniberti, G. Fagas, K. Richter), Springer, Heidelberg 2005.

[4] L. Venkataraman, J. E. Klare, C. Nuckolls, M. S. Hybertsen, M. L. Steigerwald, Nature 2006, 442, 904.

[5] M. Mayor, H. B. Weber, J. Reichert, M. Elbing, C. von Hänisch, D. Beckmann, M. Fischer, Angew. Chem. Int. Ed. 2003, 42, 5834

[6] X. Li, J. He, I. Hihath, B. Xu, S. M. Lindsay, N. I. Tao, J. Am. Chem. Soc. 2006, 128, 2135.

[7] F. Chen, X. Li, J. Hihath, Z. Huang, N. I. Tao, J. Am. Chem. Soc. 2006 128,15874 .

[8] L. Venkataraman, J. E. Klare, I. W. Tam, C. Nuckolls, M. S. Hybertsen, M. L. Steigerwald, Nano Lett. 2006, 6, 458.

[9] Y. S. Park, A. C. Whalley, M. Kamenetska, M. L. Steigerwald, M. S. Hybertsen, C. Nuckolls, L. Venkataraman, 1. Am. Chem. Soc. 2007, 129, 15768.

[10] C. A. Martin, D. Ding, J. K. Sørensen, T. Bjørnholm, J. M. van Ruitenbeek, H. S. I. van der Zant, J. Am. Chem. Soc. 2008, 130, 13198.

[11] M. Kamenetska, M. Koentopp, A. C. Whalley, Y. S. Park, M. L. Steigerwald, C. Nuckolls, M. S. Hybertsen, L. Venkataraman, Phys. Rev. Lett. 2009, 102, 126803.

[12] I. G. Kushmerick, C. M. Whitaker, S. K. Pollack, T. L. Schull, R. Shashidhar, Nanotechnology 2004, 15, 5489. 
[13] A. Danilov, S. Kubatkin, S. Kafanov, P. Hedegård, N. Stuhr-Hansen, K. Moth-Poulsen, T. Bjørnholm, Nano Lett. 2008, 8, 1.

[14] M. H. Lee, G. Speyer, O. F. Sankey, Phys. Stat. Sol. B 2006, 243, 2021.

[15] K. H. Müller, Phys. Rev. B 2006, 73, 045403.

[16] C. Li, I. Pobelov, T. Wandlowski, A. Bagrets, A. Arnold, F. Evers, F, J. Am. Chem. Soc. 2008, 130, 318.

[17] Z. Li, D. S. Kosov, Phys. Rev. B 2007, 76, 035415.

[18] S. Y. Quek, L. Venkataraman, H. J. Choi, S. G. Louie, M. S. Hybertsen, J. B. Neaton, Nano Lett. 2007, 7, 3477.

[19] I. S. Kristensen, D. ]. Mowbray, K. S. Thygesen, K. W. Jacobsen, 1. Phys.: Condens. Matter 2008, 20, 374101

[20] Y. Xue, M. A. Ratner, Phys. Rev. B 2004, 69, 085403.

[21] K. Baheti, J. A. Malen, P. Doak, P. Reddy, S.-Y. Jang, T. D. Tilley, A. Majumdar, R. A. Segalman, Nano Lett. 2008, 8, 715.

[22] J. A. Malen, P. Doak, K. Baheti, T. D. Tilley, R. A. Segalman, A. Majumdar, Nano Lett. 2009, 9, 1164.

[23] J. M. Van Ruitenbeek, A. Alvarez, I. Piñeyro, C. Grahmann, P. Joyez, M. H. Devoret, D. Esteve, C. Urbina, Rev. Sci. Instrum. 1996, 67, 108.

[24] T. Böhler, A. Edtbauer, E. Scheer, Phys. Rev. B 2007, 76, 125432

[25] T. Böhler, A. Edtbauer, E. Scheer, New J. Phys. 2009, 11, 013036.

[26] L. Grüter, M. T. Gonzălez, R. Huber, M. Calame, C. Schönenberger, Small 2005, 1, 1067.

[27] E. Lörtscher, H. B. Weber, H. Riel, Phys. Rev. Lett. 2007, 98, 176807.

[28] R. H. M. Smit, Y. Noat, C. Untiedt, N. D. Lang, M. C. van Hemert, J. M. van Ruitenbeek, Nature 2002, 419, 906.
[29] B. Xu, N. J. Tao, Science 2003, 301, 1221.

[30] S. Datta, Electronic Transport in Mesoscopic Systems, Cambridge University Press, Cambridge, UK 1997.

[31] L. Grüter, F. Cheng, T. T. Heikkilä, M. T. Gonzălez, F. Diederich, C. Schönenberger, M. Calame, Nanotechnology 2005, 16, 2143.

[32] E. H. Huisman, C. M. Guédon, B. van Wees, S. J. van der Molen, Nano Lett. 2009, 9, 3909.

[33] X. Chen, A. B. Braunschweig, M. J. Wiester, S. Yeganeh, M. A. Ratner, C. A. Mirkin, Angew. Chem. Int. Ed. 2009, 48, 5178

[34] R. Ahlrichs, M. Bär, M. Häser, H. Horn, C. Kölmel, Chem. Phys. Lett. $1989,162,165$

[35] A. Schäfer, H. Horn, R. Ahlrichs, J. Chem. Phys. 1992, 97, 2571.

[36] J. P. Perdew, Phys. Rev. B 1986, 33, 8822 .

[37] F. Pauly, J. K. Viljas, U. Huniar, M. Häfner, S. Wohlthat, M. Bürkle, J. C. Cuevas, G. Schön, New /. Phys. 2008, 10, 125019.

[38] M. S. Hybertsen, L. Venkataraman, J. E. Klare, A. C. Whalley, M. L. Steigerwald, C. Nuckolls, I. Phys.: Condens. Matter 2008, $20,374115$.

[39] L. Venkataraman, Y. S. Park, A. C. Whalley, C. Nuckolls, M. S. Hybertsen, M. L. Steigerwald, Nano Lett. 2007, 7, 502.

[40] M. ]. Ford, R. C. Hoft, A. M. McDonagh, M. B. Cortie, J. Phys.: Condens. Matter 2008, 20, 374106. 